\title{
PROPIEDADES ESTRUCTURALES, ELÉCTRICAS Y MAGNÉTICAS EN EL SISTEMA Na0.25 \\ $\mathrm{Ba}_{0.75} \mathrm{CoO}_{3}$
}

\section{Autores}

Carlos Augusto Estrada Rodriguez, Carlos Arturo Parra Vargas

Escuela de Física. Facultad de Ciencias Básicas. Grupo Física de Materiales (GFM). Universidad Pedagógica y Tecnológica de Colombia, Avenida Central del Norte, Vía Paipa, Tunja, Boyacá - Colombia.

Jorge Luiz Pimentel Junior

Instituto de Matemática, Estadística y Física, Universidade Federal do Rio Grande, Rio Grande, 96203-900.

Instituciones donde se llevó a cabo el trabajo

Universidad Pedagógica y Tecnológica de Colombia (UPTC)

Universidade Federal do Rio Grande, Rio Grande

\section{Autor responsable}

Carlos Augusto Estrada Rodriguez.

Dirección: Calle 21 № 27-37 Barrio las Lajas, Duitama (Boyacá).

Celular: 3118275925

Teléfono: 7619734

Correo Electrónico: caer8123@hotmail.com 


\title{
Resumen
}

Se reportan las propiedades estructurales, magnéticas y eléctricas del sistema $\mathrm{Na}_{0.25} \mathrm{Ba} 0.75 \mathrm{CoO}_{3}$ producido por el método de reacción en estado sólido. Los análisis de difracción de rayos X, permitieron inferir que el compuesto cristaliza en una estructura cristalina ortorrómbica de grupo espacial $C 2 / m m m$ con parámetros de red: $a=4.56 \AA, b=5.61 \AA$ y $c=10.98 \AA$. A partir de los datos de magnetización $M(T)$ y $M(H)$ se observó un comportamiento paramagnético. El ajuste de los datos de susceptibilidad permitió obtener un momento magnético efectivo de 2.17 (4) $\mu_{B}$ similar al valor del momento magnético efectivo de iones tetravalentes $\mathrm{Co}^{4+}\left(2.165 \mu_{B}\right)$ con $S=1 / 2$ en el estado de bajo espín con factor de Landé $g \approx 2.5$. A partir de la resistividad se observó un estado aislante por debajo de $100 \mathrm{~K}$, mientras que por encima de $200 \mathrm{~K}$ la resistividad presenta independencia térmica. Palabras clave: paramagnetico, estructura cristalina, momento magnético efectivo, aislante

\begin{abstract}
The structural, magnetic and electrical properties of the system $\mathrm{Na} 0.25 \mathrm{Ba} 0.75 \mathrm{CoO}_{3}$ produced by the solid state reaction method are reported. X-ray diffraction analysis allowed us to infer that the compound crystallizes in a $C 2 / \mathrm{mmm}$ orthorhombic crystal structure with network parameters: $a=4.56 \AA$, $b=5.61 \AA$ and $c=10.98 \AA$. From the magnetization data $M(T)$ and $M(H)$ a paramagnetic behavior was observed. The adjustment of the susceptibility data allowed to obtain an effective magnetic moment of 2.17 (4) $\mu_{B}$ similar to the value of the effective magnetic moment of tetravalent ions $\mathrm{Co}^{4+}$ $\left(2.165 \mu_{B}\right)$ with $S=1 / 2$ in the state of low spin with factor of Landé $g \approx 2.5$. From the resistive behavior as a function of temperature an insulation state was observed below $100 \mathrm{~K}$, while above $200 \mathrm{~K}$ the resistivity presented thermal independence.
\end{abstract}

Keywords: paramagnetic, crystalline structure, effective magnetic moment, insulation 


\section{Introducción}

La obtención de nuevos materiales ha despertado gran interés, tanto desde el punto de vista fundamental como aplicado, debido a su potencial para ser utilizados en la fabricación de dispositivos electrónicos, con mayor capacidad de almacenamiento y mayor velocidad de procesamiento (FERNANDEZ, et al, 2010). Las técnicas de caracterización, en general, han mejorado durante los últimos años, lo cual se ve reflejado en un mayor número de publicaciones relacionadas con la caracterización de todo tipo de materiales (MARTíNEZ, et al, 2013; GÓMEZ, et al, 2016). En este caso, se utilizan técnicas de caracterización como: difracción de rayos $\mathrm{X}$, medidas de magnetización en función de la temperatura, así como medidas de resistividad, entre otras.

Por otro lado, recientes investigaciones se han enfocado en las propiedades magnéticas y eléctricas asociadas a los materiales tipo cobaltita. En particular, la familia de cobaltitas de la forma La1${ }_{x} \mathrm{R}_{x} \mathrm{CoO}_{3}(\mathrm{R}=\mathrm{Ba}, \mathrm{Sr})$ ha despertado gran interés debido a la peculiar forma en que cambian sus propiedades magneto-eléctricas con la concentración del agente dopante y la temperatura. Los diagramas de fase son un referente para el análisis de las propiedades magnéticas y eléctricas de los sistemas, para estos sistemas se observó un diagrama de fases inusual en donde el comportamiento eléctrico puede coexistir entre dos diferentes estados magnéticos (MANDAL, 2004; WU, et al, 2003). Además, el aumento en la concentración Sr o Ba, permite que el sistema se convierta en un material metálico ferromagnético con momento magnético efectivo de considerables dimensiones. En particular, en los estudios de $\mathrm{La}_{1-x} \mathrm{Sr}_{x} \mathrm{CoO}_{3}$ se observó una evolución magnética hacia un estado ferromagnético de espín intermedio con electrones itinerantes $3 d$ a medida que aumenta la concentración de $\mathrm{Sr}$, mientras que para el compuesto $\mathrm{La}_{1-\times} \mathrm{Ba}_{x} \mathrm{CoO}_{3}$ se observó un comportamiento metálico tanto en el estado de ordenamiento ferromagnético como en el estado paramagnético para $x>0.2$. 
En sistemas con sustitución del átomo de lantano (SASONOV, et al, 2005), se reportó propiedades similares con respecto al comportamiento magnético.

Otro tipo de sistemas muy interesantes es la familia de cobaltitas con ausencia de dopaje de la forma: $\mathrm{ReCoO}_{3}(\mathrm{Re}=\mathrm{La}, \mathrm{Nd}, \mathrm{Gd}, \mathrm{Ho}, \mathrm{Y})$, algo relevante en los comportamientos magnéticos, excepto para los elementos $\mathrm{Nd}$ y Gd, es que no se observó transición magnética en el estado de giro a temperaturas por debajo de $300 \mathrm{~K}$ (THORNTON G, et al, 1988). 


\section{Detalles experimentales}

El sistema policritalino $\mathrm{Na} 0.25 \mathrm{Ba} 0.75 \mathrm{CoO}_{3}$ fue producido por la técnica convencional de reacción en estado sólido. La preparación se realizó a partir de la mezcla de proporciones estequiométricas de oxidos precursores $\mathrm{Na}_{2} \mathrm{O}_{2}(99.99 \%), \mathrm{BaCO}_{3}(99.8 \%) \mathrm{Co}_{3} \mathrm{O}_{4}(99.99 \%)$. El producto final se obtuvo en forma de pastilla circular, se sometió la muestra a un proceso de calcinado a $600{ }^{\circ} \mathrm{C}$ durante 36 horas, además, se realizaron dos tratamientos térmico de sinterización, uno a $620^{\circ} \mathrm{C}$ durante 24 horas y el otro a $640{ }^{\circ} \mathrm{C}$ durante un tiempo de 40 horas de las cuales 30 horas fueron con suministro de oxigeno. Las medidas de difracción de rayos $\mathrm{X}$ se realizaron utilizando un difractómetro comercial de rayos X de Cu (radiación Ka) y longitud de onda 1.5405Å utilizando la geométrica Bragg-Brentano (WOOLFSON M, 1997), se midió con un paso de $0.02^{\circ}$ en el rango de 20 a 90 grados con un tiempo de exposición de 2 seg. El refinamiento del patrón de difracción se obtuvo a través del método Rietveld utilizando el programa GSAS de interfaz gráfica EXPGUI (LARSON and DREELE, 2004). Las medidas de magnetización fueron ralizadas con un magnetómetro de SQUID modelo MPMS-XL de la empresa Quantum Design Inc midiendo magnetización en función de la temperatura $(M X T)$ siguiendo los procedimientos de enfriamiento en presencia de campo FC (Field Cooled) y enfriamiento sin campo ZFC (Zero Field Cooled) de $5 \mathrm{~K}$ a $300 \mathrm{~K}$ aplicando campo magnético de 50 Oe, y magnetización en función de campo magnético $(M X H)$ con evaluación de isotermas cerca de la temperatura de Curie.

Las medidas de resistividad se realizaron en una región rectángular de la muestra $(2.9 \times 0.95 \times 1.72$ $\mathrm{mm}^{3}$ ) utilizando la técnica experimental de cuatro puntas con ausencia de campo magnético en un rango de $5 \mathrm{~K}$ a $300 \mathrm{~K}$ aplicando densidad de corriente de $0.5 \mathrm{~mA}$. 


\section{Resultados y discusión}

El patrón experimental de difracción de rayos $\mathrm{X}$ del sistema $\mathrm{Na} 0.25 \mathrm{Ba} 0.75 \mathrm{CoO}_{3}$ se puede observar en la Figura 1, se presenta una doble fase la cual fue corroborada e identificada por medio del refinamiento. La Figura 2 muestra el refinamiento Rietveld del patrón de difracción de rayos X. La línea roja corresponde al patrón teórico de ajuste usando el software GSAS, las cruces representan el patrón experimental. La línea verde representa el background y la línea azul es la diferencia entre los patrones experimental y teórico. Los parámetros de red y posiciones atómicas obtenidos con el refinamiento Rietveld se muestran en la tabla 1. A partir del análisis de refinamiento Rietveld en la muestra $\mathrm{Na} 0.25 \mathrm{Ba} 0.75 \mathrm{CoO}_{3}$, fue posible identificar la presencia de dos fases, la fase mayoritaria de grupo espacial $C 2 / \mathrm{mmm}$, y la fase secundaria de grupo espacial $P 6 / \mathrm{mmm}$.

Las Figuras 3 y 4 muestran las micrografías eléctricas de barrido del sistema $\mathrm{Na}_{0.25} \mathrm{Ba}_{0.75} \mathrm{CoO}_{3}$. Se puede observar una microestructura fina y más granular con el aumento del índice donde los granos poseen una forma de aguja con una aparente densificación estructural.

La evolución térmica de la susceptibilidad magnética molar del sistema $\mathrm{Na}_{0.25} \mathrm{Ba}_{0.75} \mathrm{CoO}_{3}$ medida con campo magnético de 50 Oe se muestra en la Figura 5. Se observa un aumento en la susceptibilidad a medida que diminuye la temperatura acorde a un comportamiento de tipo paramagnético. Esta curva fue ajustada usando la ley de Curie-Weiss $\left(\chi=\chi_{0}+\mathrm{C} / T-\theta\right)$, donde $\chi_{0}$ es la susceptibilidad independiente de la temperatura, $\mathrm{C}$ es la constante de Curie, y $\theta$ la temperatura de Weiss. Los valores obtenidos fueron: $\mathrm{C}=0.592 \mathrm{emu} \mathrm{K} / \mathrm{Oe} \mathrm{mol}, \theta=16.8 \mathrm{~K} \mathrm{y} \chi_{0}=4.87 \times 10^{-4} \mathrm{emu}^{\mathrm{mol}}{ }^{-1}$. El valor positivo de $\theta$ indica interacción de tipo ferromagnético entre momentos magnéticos de cobalto.

A partir del ajuste de la ley de Cuie Weiss en la curva $\chi^{-1}$ se obtuvo un momento magnético efectivo de 2.17 (6) $\mu_{B}$, este valor es similar a lo reportado en iones tetravalentes $\mathrm{Co}^{4+}$ con $S=1 / 2$ y factor de Landé $g \approx 2.5$ donde la contribución magnética es $2.2 \mu_{B}$ (CHOU F. C, Cho J. H and Lee Y. S, 2004). 
Ademas, se observa una desviación lineal por encima de $100 \mathrm{~K}$ asociada a un incremento en la interaccion magnetica, en otros tipo de cobaltitas se observó un comportamiento similar por debajo de $150 \mathrm{~K}$ (MIKAMI M, et al, 2003).

La magnetización en función del campo magnético se muestra en la Figura 6 . El sistema exhibe un comportamiento lineal de la magnetización a medida que se incrementa el campo magnético en direccion positiva, de manera similar este comportamiento se presenta en direccion negativa de campo magnético. Esto está en concordancia con el comportamiento observado en curvas de $M(H)$ para materiales paramagnéticos (ELFRESH Mc M, 1994), ademas, la curva de magnetización en función de la temperatura corrobora el estado paramagnético en el sistema $\mathrm{Na} 0.25 \mathrm{Ba} 0.75 \mathrm{CoO}_{3}$. Por otro lado, se observa un cambio en el comportamiento magnético a través de la disminución en la magnetización a medida que aumenta la temperatura.

La resistividad en función de la temperatura, medida en ausencia de campo magnético se muestra en la Figura 7. Por encima de $200 \mathrm{~K}$, se observó que la resistividad presenta un comportamiento casi independiente de la temperatura, mientras que por debajo de $100 \mathrm{~K}$ se incrementa rápidamente a medida que disminuye la temperatura, este comportamiento eléctrico es típico de los materiales tipo aislante. Ademas, el inusual estado eléctrico es cualitativamente similar a lo observado por otros autores que estudiaron el comportamiento resistivo de películas compuestas de niquel en condiciones de baja concentración de cobalto (ANDROULASKI J, et al, 2004). 


\section{Conclusion y Recomendaciones}

El refinamiento del patrón de difracción de rayos $\mathrm{X}$, permitió demostrar que el compuesto $\mathrm{Na} 0.25 \mathrm{Ba} 0.75 \mathrm{CoO}_{3}$ cristaliza en una estructura cristalina tipo ortorrómbica con fase mayoritaria de gru-

po espacial $C 2 / \mathrm{mmm}$ y parámetros de red $a=4.56 \AA, b=5.60 \AA, c=10.98 \AA$. Se observó un comportamiento paramagnético donde la contribución magnética del momento magnéticos efectivo es $1.73 \mu_{B}$. En la región de altas temperaturas $(T>200 \mathrm{~K}$ ), se observó un comportamiento resistivo casi independienten de la temperatura, mientras que en la región $\mathrm{T}<100 \mathrm{~K}$ el sistema presentó un estado tipo aislante.

De los resultados de este trabajo de investigación se espera que tengan un aporte significativo y de gran utilidad, ademas, que sea un referente para posteriores procesos de investigación y aplicación tecnológica de los nuevos materiales enmarcados en el estudio de altas y bajas temperaturas en temas afines con las Ciencias - Física. 


\section{Agradecimientos}

A la Universidad Pedagógica y Tecnológica de Colombia (UPTC) y en particular al grupo de investigación de física de materiales en cabeza del Dr. Carlos Arturo Parra Vargas por su apoyo en gran medida en todo el proceso experimental, al Instituto de Matemática, Estadística y Física, Universidade Federal do Rio Grande, Rio Grande, particularmente al Dr. Jorge luiz Pimentel Junior por las medidas de magnetización, resistividad y sus aportes significativos en el análisis de resultados. 


\section{Referencias bibliográficas}

[1] Fernández F H, Duarte, J E, Samitier-Marti, J. (2010). Manipulación de micropartículas por medio de una microestructura de electrodos en espiral. Revista de Investigación, Desarrollo e Innovación, 1 (1), 70-79. Recuperado de:

http://uptc.metarevistas.org/index.php/investigacion_duitama/article/view/1295

[2] Gómez O, Díaz-Lagos, M, Blandón-Montes, A., \& Martínez-Ovalle, S. (2016). Presencia de elementos contaminantes como $\mathrm{Cd}, \mathrm{As}, \mathrm{Pb}$, Se y $\mathrm{Hg}$ en carbones de la zona Cundiboyacense, Colombia. Revista de Investigación, Desarrollo e Innovación, 7(1), 141-150. doi:

http://dx.doi.org/10.19053/20278306.v7.n1.2016.5604

[3] Martínez-Ovalle, S, Reyes-Caballero, F., \& González-Puin, L. X. (2013). Protección radiológica a trabajadores y público en instalaciones que operan radioisótopos industriales. Revista de Investigación, Desarrollo e Innovación, 3 (2), 120-124. doi: 10.19053/20278306.2166

[4] Mandal P, Choudhury P, Biswas S. K. and Ghosh B, (2004). Transport and magnetic properties of $\mathrm{La}_{1-x} \mathrm{Ba}_{x} \mathrm{CoO}_{3}$, Phys. Rev. B, 70:104407.

[5] Wu J and Leighton C, (2003). Glassy ferromagnetism and magnetic phase separation in La1${ }_{x} \mathrm{Sr}_{x} \mathrm{CoO}_{3}$, Phys. Rev. B, 67: 174408.

[6] Sazonov A P, Troyanchuk I O, Sikolenko V V, Chobot G M and Szymczak H, (2005). Crystal structure, magnetic and electrical properties of the $\mathrm{Nd}_{1-x} \mathrm{Ba}_{x} \mathrm{CoO}_{3}$ system. Journal of Physics: Condensed matter, 17: 1

[7] Thornton G, Morrison F C, Partington S, Tofield B C, Williams D E, (1988). The rare earth cobaltates: localised or collective electron behaviour?, Journal Physic C, Solid State Phys, 21: 2871 [8] Woolfson M, (1997). An Introduction to X-ray Crystallography, Second Edition. 
[9] Larson A.C. and Von Dreele R.B, (2004). General Structure Analysis System (GSAS), Los Alamos National Laboratory Report LAUR, 86-748.

[10] Chou F. C, Cho J. H and Lee Y. S, (2004). Magnetic susceptibility study of hydrated and nonhydrated $\mathrm{Na}_{x} \mathrm{CoO}_{2}$ y $\mathrm{H}_{2} \mathrm{O}$ single crystals, Phys. Rev B, 70: 144526.

[11] Mikami M, Yoshimura M, Mori Y, Sasaki T, Funahashi and M. Shikano, (2003). Thermoelectric Properties of Two $\mathrm{Na}_{x} \mathrm{CoO}_{2}$, J. Appl. Phys. 42: 7383-7386.

[12] Mc Elfresh M, (1994). Fundamentals of magnetism and magnetic measurements. Featuring Quantum Design's magnetic property measurement system. Quantum Design.

[13] Androulakis J, Klini A, Manousaki A, Violakis G, Giapintzakis J, (2004). Growth of polycrystalline $\mathrm{LaNi}_{1-x} \mathrm{Co}_{x} \mathrm{O}_{2}(x=0.3,0.5)$ thin films on Si (100) by pulsed laser deposition, Appl. Phys. A, 79: 671 675. 
Tabla 1. Parámetros de red, parámetros de ajuste y posiciones atómicas de $\mathrm{Na}_{0.25} \mathrm{Ba}_{0.75} \mathrm{CoO}_{3}$

\begin{tabular}{|c|c|c|c|c|c|c|c|}
\hline Fase 1 & & po espacial & $\mathrm{C} 2 / \mathrm{mmm}$ & Fase 2 & & Grupo esp & acial $P 6 / \mathrm{mmm}$ \\
\hline$a=4.56 \AA$ & $b=5.61 \AA$ & $C=10.98 \AA$ & $\mathrm{Vol}=280.8 \AA^{3}$ & $a=b=$ & $5.6 \AA$ & $C=4.76 \AA$ & $\mathrm{Vol}=129.7 \AA^{3}$ \\
\hline & & & POSICIONES A & DMICAS & & & \\
\hline Atomos & $x$ & $y$ & $z$ & Atomos & $x$ & $y$ & $z$ \\
\hline $\mathrm{Na}$ & -0.75 & 5.09 & 2.74 & $\mathrm{Ba}_{1}$ & 0.66 & 0.33 & 0 \\
\hline $\mathrm{Ba}$ & 0.21 & 4.24 & 0.48 & $\mathrm{Ba}_{2}$ & 0.33 & 0.66 & 0.5 \\
\hline Co & 1 & 0 & 0 & $\mathrm{Co}_{1}$ & 0 & 0 & 0.26 \\
\hline 0 & 0.84 & -9.74 & 0.75 & $\mathrm{O}_{1}$ & 0.14 & 0.85 & 0 \\
\hline & & & & $\mathrm{O}_{2}$ & 0.83 & 0.16 & 0.5 \\
\hline
\end{tabular}

$\mathrm{R}=0.17 \quad \chi^{2}=2.95$ 


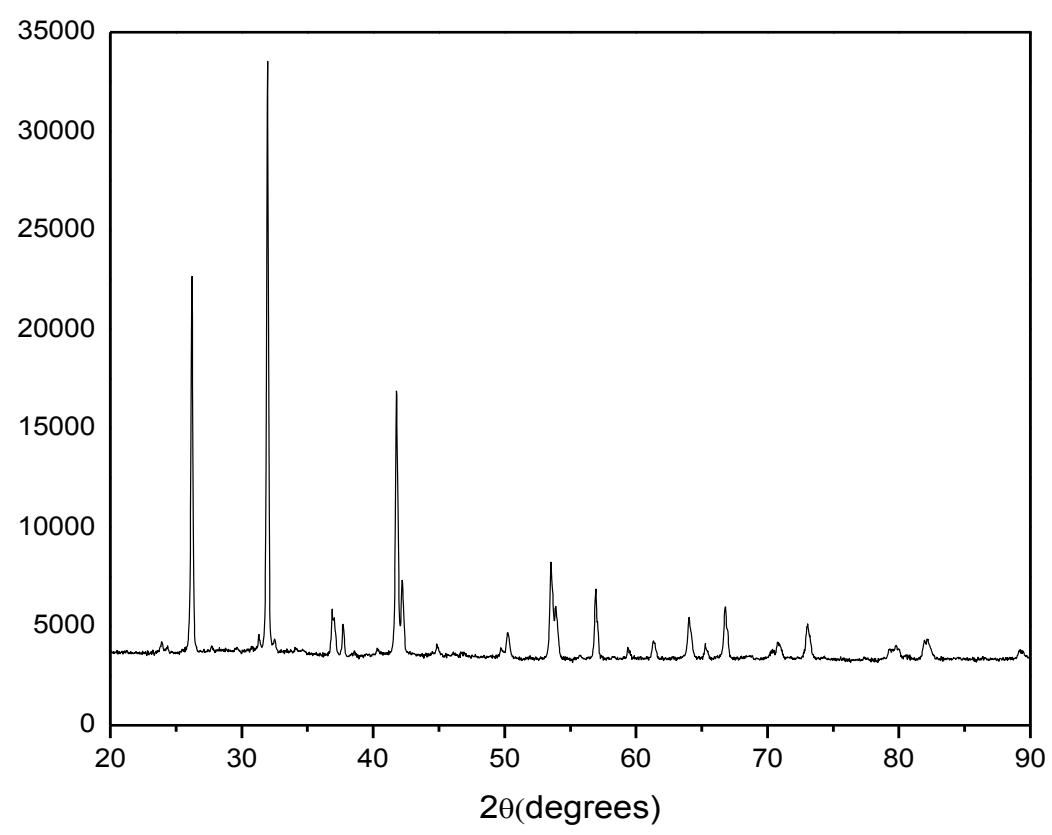

FIG. 1. Patrón de difracción de rayos $\mathrm{X}$ registrados con radiación Cu Ka.

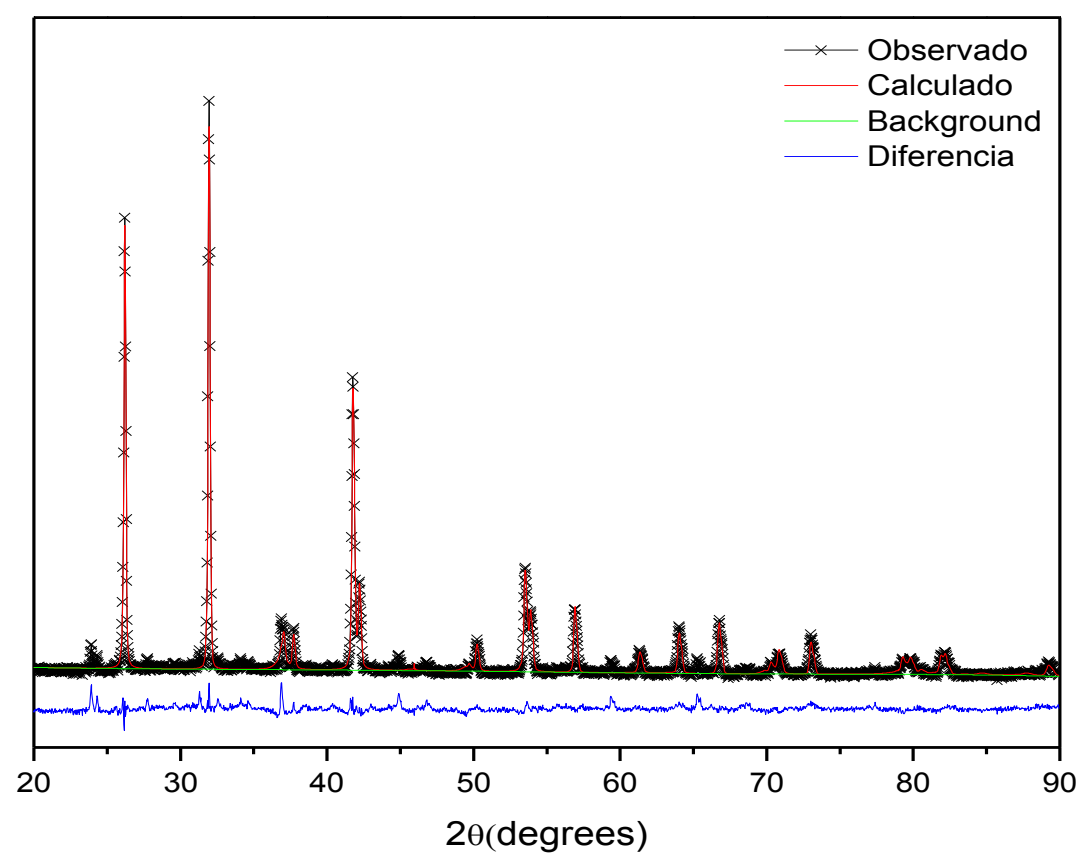

FIG. 2. Refinamiento Rietveld de la muestra $\mathrm{Na}_{0.25} \mathrm{Ba} 0.75 \mathrm{CoO}_{3}$ 


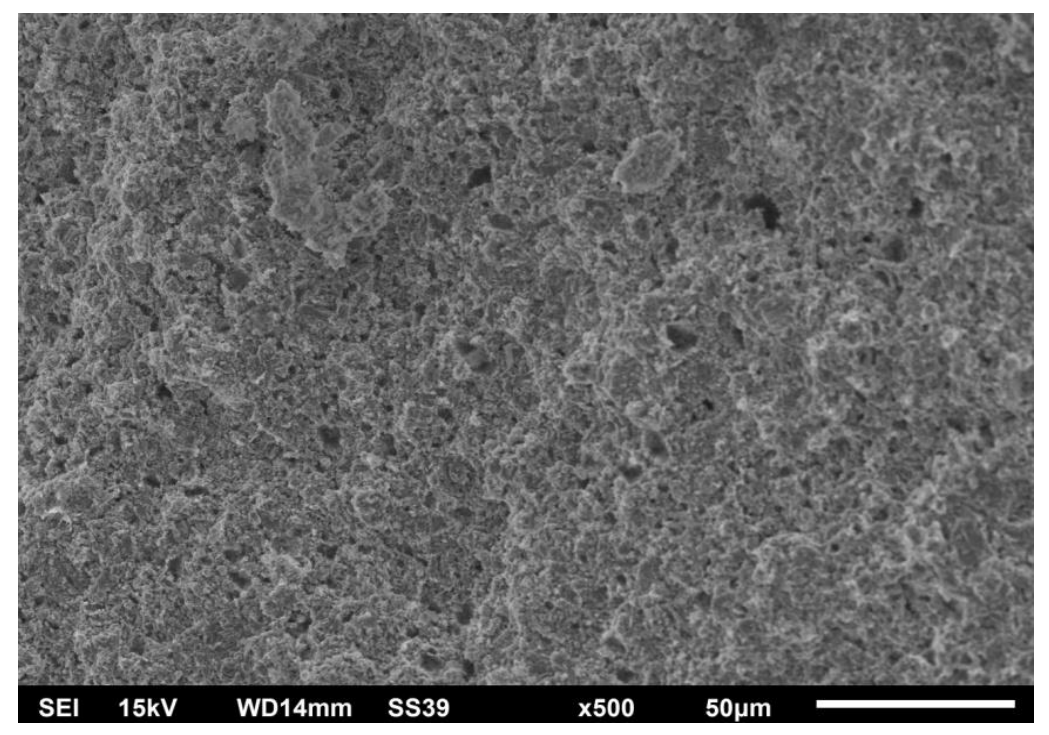

FIG. 3 Micrografias SEM con tamaño de grano de $50 \mu \mathrm{m}$

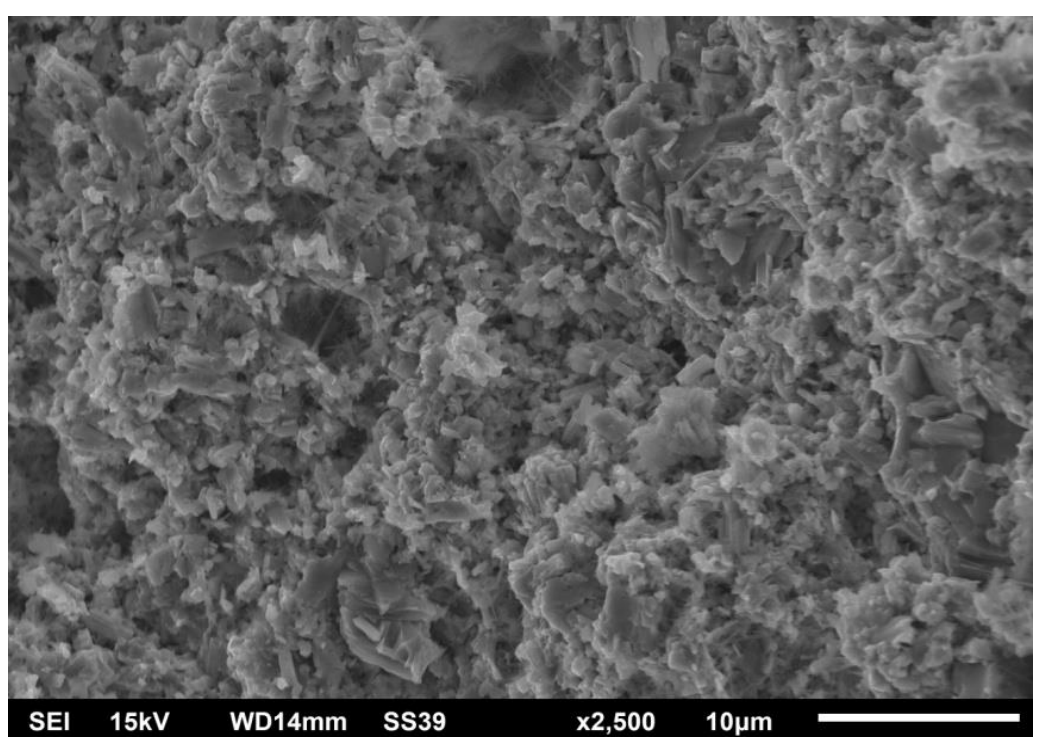

FIG. 4 Micrografias SEM con tamaño de grano de $10 \mu \mathrm{m}$ 


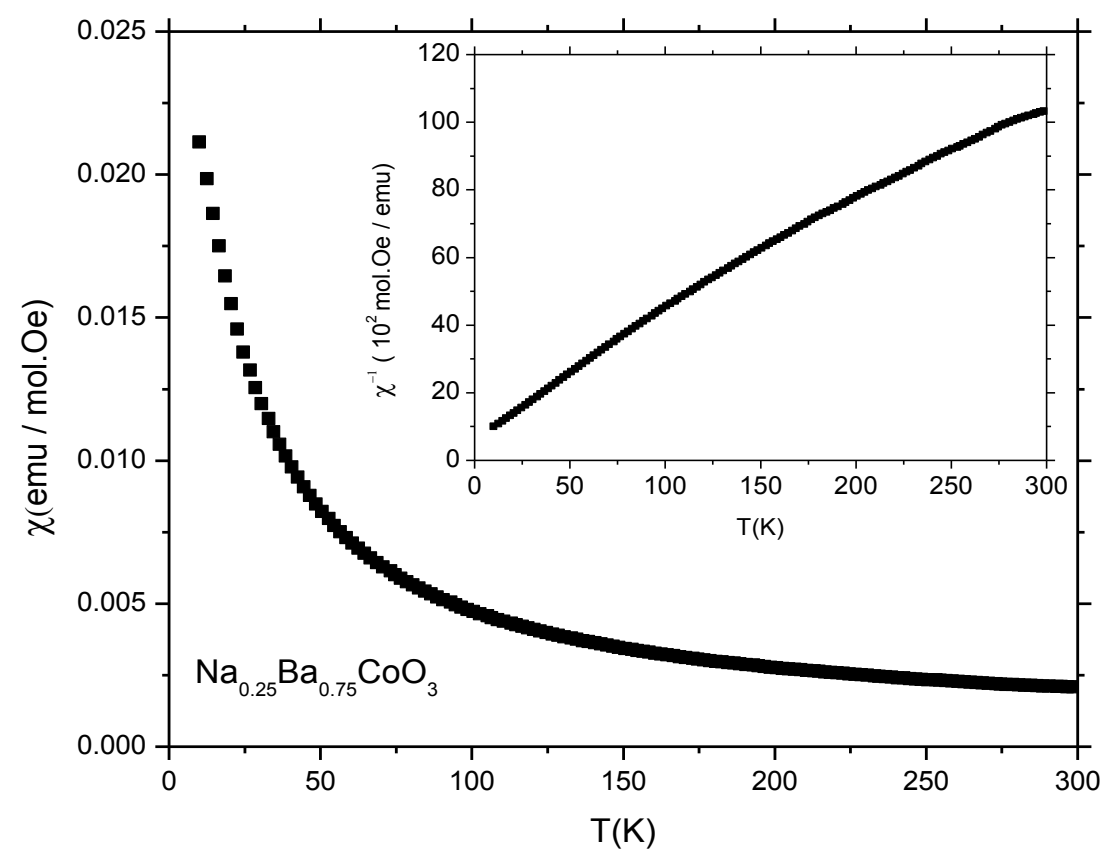

FIG. 5. Susceptibilidad molar en modo ZFC. El inset de la figura representa la evolución térmica de la susceptibilidad molar inversa con la temperatura.

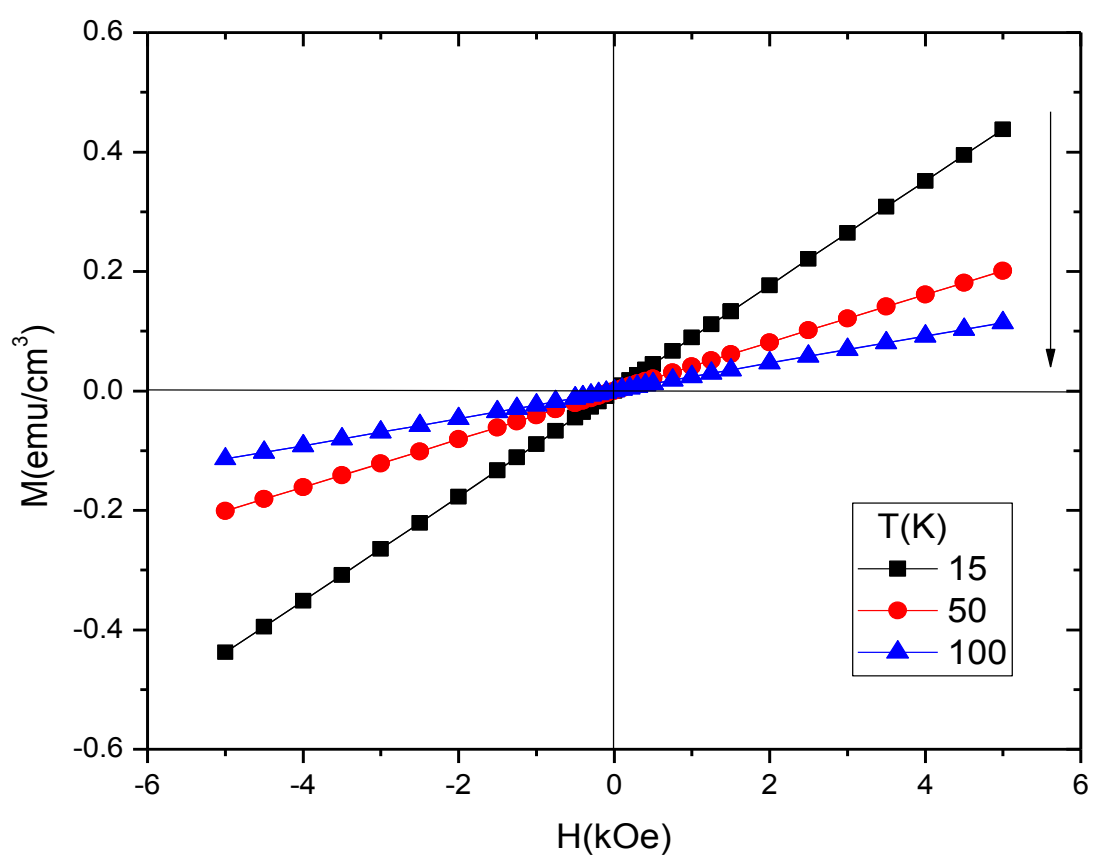

FIG. 6. Curvas de magnetización con dependencia de campo magnético de $\mathrm{Na}_{0.25} \mathrm{Ba}_{0.75} \mathrm{CoO}_{3}$. 


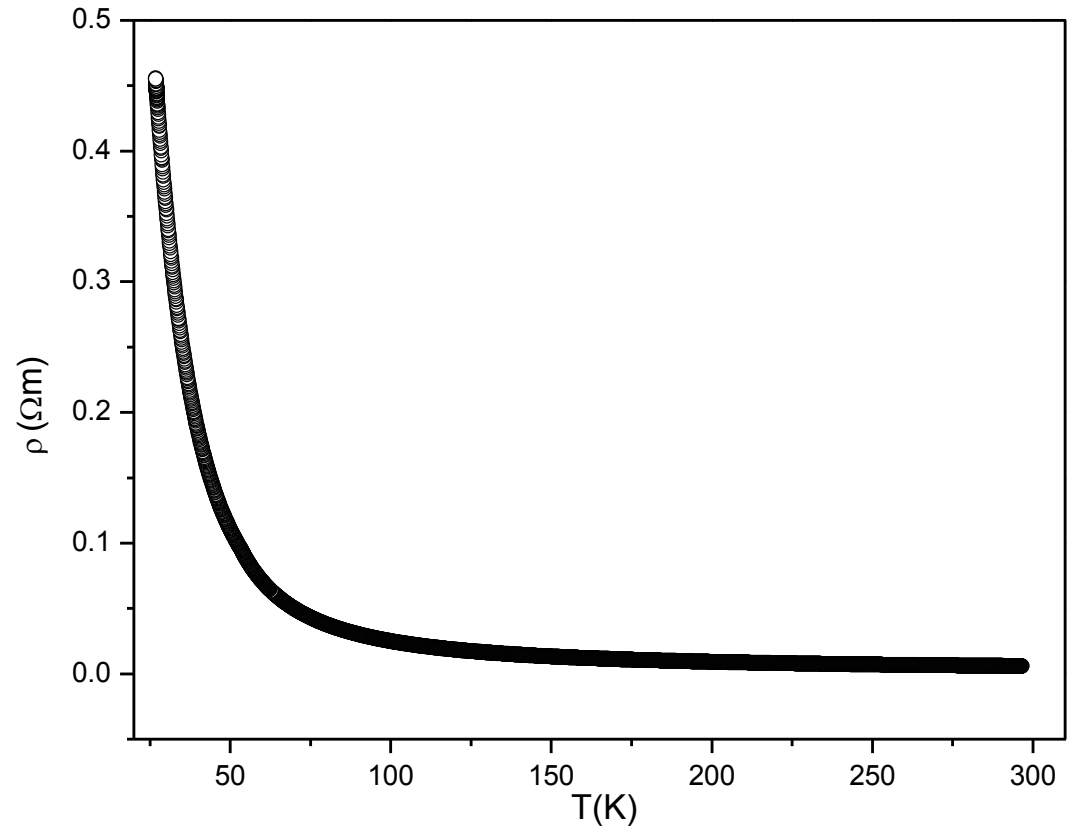

FIG. 7. Curva de resistividad en función de la temperatura de $\mathrm{Na} 0.25 \mathrm{Ba} 0.75 \mathrm{CoO}_{3}$. 